
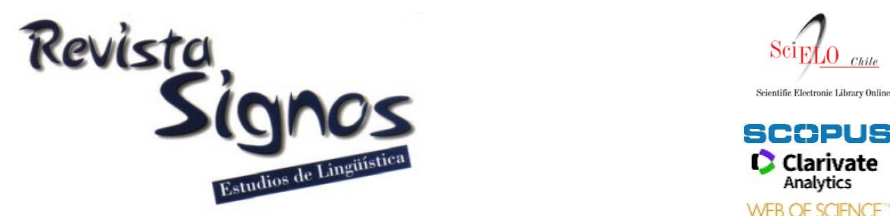

\title{
"Esta batalla la tenía que ganar": La metáfora, modalidad y el género en narrativas de cáncer*
}

\section{"I had to win this battle": Metaphor, modality and gender in cancer narratives}

\author{
Dalia Magaña \\ UNIVERSIDAD DE CALIFORNIA EN MERCED \\ ESTADOS UNIDOS \\ dmagana6@ucmerced.edu
}

\author{
Teenie Matlock \\ UNIVERSIDAD DE CALIFORNIA EN MERCED \\ ESTADOS UNIDOS \\ tmatlock@ucmerced.edu
}

Recibido: 19-IX-2019 / Aceptado: 28-X-2020

DOI: $10.4067 /$ S0718-09342021000100191

\section{Resumen}

Los estudios de análisis de discurso ofrecen herramientas para revelar, desde la perspectiva del paciente, cómo se entienden enfermedades complejas como el cáncer. Aunque varios estudios han arrojado luz sobre cómo se usa la metáfora y la modalidad en el discurso del cáncer en inglés, poco se conoce sobre el discurso sobre el cáncer en español. Para descubrir cómo los pacientes hispanohablantes hablan naturalmente sobre sus experiencias personales con el cáncer y cuáles diferencias de género existen en el discurso sobre el cáncer realizamos un estudio de corpus de 50 narraciones escritas en español. Las narraciones provienen de un sitio en línea dedicado a compartir experiencias personales con la enfermedad. Analizamos cómo 25 hombres y 25 mujeres usan la metáfora y la modalidad en las narrativas sobre el cáncer. Nuestro hallazgo fue que las metáforas de guerra y metáforas de trayectoria predominan en narraciones de salud. Notamos que los escritores usaron metáforas de guerra para ofrecer consejos a los lectores y para retratar al cáncer como un enemigo. Usaban metáforas de trayectoria para discutir su progreso y para ofrecer aliento a sus lectores. Descubrimos que las mujeres tienden a usar metáforas contra el cáncer con más frecuencia que los hombres. Finalmente, encontramos que tanto la frecuencia como los tipos de verbos de modalidad que usan evidencian la inclinación de las mujeres a establecer conexiones y la tendencia de los hombres a ofrecer y buscar información.

Palabras Clave: Discurso de salud, teoría de la metáfora conceptual, modalidad, género, narrativas de cáncer. 


\begin{abstract}
Discourse analysis studies offer tools to reveal, from patients' perspective, how complex diseases such as cancer are understood. Although several studies have shed light on how metaphor and modality are used in cancer discourse in English, little is known about cancer discourse in Spanish. To discover how Spanish-speaking patients narrate their own personal experiences with cancer and what gender differences exist in cancer discourse, we compiled a corpus of 50 Spanish narratives. The narratives were collected from an online site dedicated to sharing personal experiences with the disease. We analyze how 25 men and 25 women use metaphor and modality in cancer narratives. Our main finding is that violence metaphors and travel metaphors predominate in health narratives. We note that writers used metaphors of violence to offer readers advice and to portray cancer as an enemy. They used travel metaphors to discuss their progress and to offer encouragement to their readers. We found that women tend to use cancer metaphors more often than men. Finally, we find that both the frequency and the types of modal verbs they use demonstrate the inclination of women to establish connections and the tendency of men to offer and seek information.
\end{abstract}

Key Words: Health discourse, conceptual metaphor theory, modality, gender, cancer narratives.

\title{
INTRODUCCIÓN
}

Cuando a las personas se les diagnostica con cáncer, experimentan miedo, frustración y desesperación. Hay trabajos de investigación que atribuyen estos sentimientos negativos a diversos factores que incluyen la falta de compresión de la enfermedad, los riesgos asociados con los tratamientos y la confusión sobre el papel de los médicos en el tratamiento (Stark \& House, 2000; Raison \& Miller, 2003). Además, es un desafío para los pacientes con cáncer y para los médicos comunicarse de manera efectiva sobre la enfermedad (Sontag, 1989; Epstein, Prigerson, O’Reilly \& Maciejewski, 2016). Un problema es que los médicos sobreestiman sus habilidades para hablar sobre el cáncer con sus pacientes (Ha \& Longnecker, 2010). Para obtener más información sobre cómo los pacientes hablan naturalmente sobre sus propias experiencias personales con el cáncer, realizamos un estudio de corpus sobre cómo los hombres y las mujeres usan la metáfora y la modalidad en las narrativas sobre el cáncer. Nos enfocamos en cómo los pacientes con cáncer de habla hispana se comunican en un sitio en línea dedicado a compartir experiencias personales con la enfermedad.

Antes de describir algunos detalles del estudio, posicionaremos el uso de la metáfora y la modalidad en el discurso sobre el cáncer. En primer lugar, la metáfora es omnipresente en el lenguaje cotidiano. Se usa para hablar sobre muchos temas diferentes, por ejemplo, el tiempo (Clark, 1973; Moore, 2006), las matemáticas (Lakoff \& Núñez, 2000), la física (Brookes \& Etkina, 2007), el internet (Matlock, Castro, Fleming, Gann \& Maglio, 2014), las relaciones personales (Winter \& Matlock, 2013), la economía (Henderson, 1982), la política (Lakoff, 2008), las emociones (Kövecses, 
2003), el crimen (Thibodeau \& Boroditsky, 2011), y las enfermedades y sus tratamientos (Skott, 2002; Semino, Demjén, Demmen, Koller, Payne, Hardie \& Rayson, 2015; Williams, 2016). La metáfora permite hablar de algo abstracto en términos relativamente más concretos (Lakoff \& Johnson, 1980; Gibbs, 1994; Kövecses, 2008). Por ejemplo, la metáfora de trayectoria o de carrera son comunes en discursos políticos (Duarte, 2016; Matlock, 2013). En el discurso político de Hugo Chávez se retrata al socialismo como una trayectoria a la cual se hace referencia por medio de palabras que señalan una ubicación, un movimiento y una meta: “... aquí estamos en presencia real y concreta del nuevo socialismo que vamos construyendo paso a paso como única vía para salir del atraso" (Duarte, 2016: 39). Notemos que en este ejemplo se alude a las dificultades en el movimiento en esta trayectoria o vía por medio del uso de 'salir' y 'atraso', lo que implica que hay barreras o una fuerza de resistencia que afectan al movimiento.

Los académicos de diversos campos, incluidos la lingüística y la psicología, han llegado a la conclusión de que la metáfora es fundamental para la forma en que las personas llegan al razonamiento sobre los estados y las acciones en el mundo (Lakoff \& Johnson, 1980; Gibbs, 1994; Kövecses, 2005, 2015; Lakoff, 2008; Thibodeau \& Boroditsky, 2011).

En segundo lugar, el análisis de metáforas nos informa sobre cómo la sociedad retrata a grupos de personas y sus culturas y lenguas. Por ejemplo, los medios populares han retratado al español como una 'barrera' para los latinos en los Estados Unidos (Santa Ana, 2002; Achugar, 2008). Como señaló Koller (2004), a las mujeres empresarias a menudo se les describe metafóricamente como guerreras o luchadoras en las revistas populares, con mucha más frecuencia que a los hombres de negocios. La autora explica que la atribución a las mujeres de rasgos a menudo atribuidos a los hombres hace que sean aceptadas como miembros del grupo con igual status que los hombres en una esfera socioeconómica definida y dominada por éstos.

En tercer lugar, las metáforas de guerra a menudo aparecen en el discurso popular sobre el cáncer. En uno de sus ejemplos, Williams (2016: 123) cita a un periódico español que usa una metáfora de la guerra que sirven para ilustrar un enfrentamiento:

"Con el conocimiento que se tenía de la célula hasta hace poco se podía atacar al cáncer a cañonazos: tanto la quimioterapia como la radioterapia matan las células malignas pero también a las sanas."

Es decir, lidiar con el cáncer es una lucha física y la medicación es un arma. Tal lenguaje muestra cómo el discurso moviliza la violencia para describir varios aspectos de un diagnóstico de cáncer, incluyendo cómo la enfermedad y el tratamiento afectan el cuerpo y cómo los humanos lidian con la enfermedad y el tratamiento. En este trabajo, consideramos varios tipos de metáforas, incluidas aquellas que son más 
militaristas y aquellas que aluden a un enfrentamiento, dentro de la categoría de metáforas de guerra. Tal lenguaje surge en la comunicación entre pacientes y médicos, las interacciones entre los pacientes, en la literatura médica y farmacéutica, y en los medios populares.

En los últimos tiempos, un número creciente de académicos, pacientes y doctores han criticado el uso de metáforas de la guerra en la comunicación del cáncer. Algunos abogan por la eliminación del lenguaje sobre la guerra, incluido el lenguaje figurativo, especialmente con o sobre pacientes con cáncer (Sontag, 1989; Laranjeira, 2013; Hurley, 2014; Domínguez \& Sapiña, 2016). Una de las razones es que los pacientes pueden encontrar ofensivas las referencias a la guerra, y otra es que pueden inducir a los pacientes de cáncer a suponer que la enfermedad tiene un poder o una capacidad mayor de la que realmente tiene (Reisfield \& Wilson, 2004). El lenguaje de la guerra también puede hacer que algunos pacientes con cáncer se sientan victimizados o aislados (Sontag, 1989; Rojas Miranda \& Fernández González, 2015).

En su influyente ensayo Sontag (1989) explica que las metáforas de guerra evocan un sentimiento de victimización, porque también se han usado en el dominio de otros problemas sociales como en la guerra contra las drogas / obesidad / pobreza. Sontag (1989) no quería asociarse a sí misma o a su enfermedad con estas guerras sociales y argumenta que otras personas con cáncer pueden sentir lo mismo. Otros trabajos de investigación sugieren que las metáforas de la guerra pueden ser útiles para la comunicación del cáncer. Skott (2002), por ejemplo, afirma que enmarcar metafóricamente el cáncer como un enemigo para luchar y conquistar conduce a un sentimiento de solidaridad y comunidad entre los pacientes con cáncer, que ganan confianza para compartir experiencias con otras personas afectadas por el cáncer. También se ha reconocido que las metáforas de la guerra (y las de trayectoria) pueden hacer que los pacientes con cáncer se sientan empoderados (Bowker, 1996; Hurley, 2014). Además de observaciones existentes, un estudio afirma que las interacciones entre médicos y pacientes son más efectivas cuando usan metáforas (Casarett, Pickard, Fishan, Alexander, Arnold, Pollak \& Tulsky, 2010).

En nuestro propio análisis de las metáforas, partimos desde el trabajo previo sobre la metáfora en el discurso del cáncer, especialmente la investigación de Gibbs y Franks (2002) y Semino et al. (2015), quienes informaron que los pacientes con cáncer frecuentemente usan metáforas de la guerra y metáforas de trayectoria. Estos y otros estudios han proporcionado información valiosa sobre cómo y cuándo los pacientes usan la metáfora. Si bien muestran claramente que las metáforas pueden empoderar y desapoderar a los pacientes con cáncer, se necesita más investigación para comprender mejor cómo los pacientes usan la metáfora para hablar sobre sus propias experiencias con el cáncer y lo que eso refleja sobre sus concepciones básicas de la enfermedad. Además, se debe prestar atención a cómo se usan las metáforas en varios idiomas y culturas. Hasta la fecha, la investigación sobre cómo se usan las metáforas en la 
comunicación del cáncer se ha centrado casi exclusivamente en el inglés. Al examinar la metáfora en otros idiomas, podemos aprender más acerca de qué metáforas surgen naturalmente cuando las personas se comunican sobre el cáncer, y qué metáforas pueden ser más útiles para los pacientes, en última instancia. Es importante tener en cuenta diversos contextos lingüísticos y culturales al analizar la comunicación sobre asuntos sociales.

En Magaña y Matlock (2018), se analizaron entradas de blog de pacientes con cáncer de habla hispana. Descubrimos que las metáforas que utilizaron son consistentes con metáforas utilizadas por pacientes con cáncer de habla inglesa. La mayoría de los blogs analizados en ese estudio fueron generados por mujeres. El estudio actual amplía ese trabajo centrándose en las diferencias de género en el discurso sobre el cáncer. Esta comparación es importante porque los hombres y las mujeres tienen diferentes presiones sociales y hablan de enfermedades de manera diferente. Los estudios de hombres y mujeres de habla inglesa, sugieren que cuando los hombres hablan sobre el cáncer de próstata, lo hacen para buscar o compartir información sobre el cáncer. Por el contrario, cuando las mujeres hablan sobre el cáncer de mama, a menudo transmiten aliento y apoyo a los demás (Klemm, Hurst, Dearholt \& Trone, 1999; Kiss \& Meryn, 2001; Seale, Ziebland \& Charteris-Black, 2006; Gooden \& Winefield, 2007). Las mujeres también son más propensas a confiar en los demás que los hombres (Harrison, Maguire \& Pitceathly, 1995).

Los hombres y las mujeres no solo se centran en diferentes temas en su discurso sobre el cáncer, sino que también los transmiten usando distintos recursos lingüísticos. Un recurso lingüístico importante en el contexto médico que ofrece conocimiento sobre estas diferencias es el uso de la modalidad (Charteris-Black \& Seale, 2010). En su estudio, Charteris-Black y Seale (2010) analizan cómo los hombres y las mujeres hablan sobre una variedad de enfermedades, incluidas la depresión, el cáncer y las enfermedades del corazón. Sus datos consisten en casi 200 entrevistas enviadas a un sitio web de salud del Reino Unido. Uno de sus hallazgos es que las mujeres usan más recursos de modalidad que los hombres. Las mujeres cambian entre la modalidad baja (por ejemplo, 'pienso que', 'creo que', 'me imagino') y la modalidad alta (por ejemplo, 'sé', 'necesito'). Según el estudio, las mujeres se sienten inclinadas a utilizar una modalidad baja para expresar incertidumbre sobre sus enfermedades y una modalidad alta para expresar sus experiencias de salud y ofrecer consejos.

Nuestro estudio examina la metáfora y la modalidad en las narrativas en línea de pacientes y sobrevivientes de cáncer de habla hispana. De manera semejante a como los hablantes usan metáforas conceptuales para referirse indirectamente al cáncer, la modalidad ofrece recursos para hacer afirmaciones en una manera indirecta, semejante a cómo. Los recursos para expresar modalidad son multifuncionales ya que las personas los usan para expresar vacilación, solidaridad, cortesía, respeto y/o humildad 
(Hyland, 1996). La investigación sobre la modalidad en las interacciones médicas nos ayuda a entender la negociación de una relación interpersonal entre pacientes y médicos, incluidas, las estrategias de cortesía, la negociación de roles sociales y de poder, y expectativas y valores culturales; aspectos del habla que son importantes en la comunicación sobre problemas de salud (Caffi, 1999; Delbene, 2004; Cordella, 2007; Flores-Ferrán, 2010, 2012). Los hablantes usan la modalidad para evitar riesgos innecesarios, responsabilidades y conflictos (Caffi, 2007) y en el contexto médico puede revelar información importante.

Un estudio de las interacciones médico-paciente en español encuentra que los pacientes usan la modalidad cuando revelan detalles íntimos sobre su salud mental o cuando le brindan información médica al médico (Magaña, 2017). En ese estudio se sugiere que la ambigüedad que la modalidad crea puede facilitar que los pacientes ofrezcan detalles con cautela sin que se los responsabilice por la información. Por ejemplo, al describir sus síntomas de ansiedad un paciente usa la modalidad verbal, 'Siempre parece que me estoy ahogando', para describir su experiencia sin estar comprometido a la exactitud de la información (Magaña, 2017). En este estudio, los pacientes también usan la modalidad para mostrar deferencia al médico. El médico usa la modalidad para establecer confianza, mitigar su estado de poder y desplazar la responsabilidad de los pacientes.

El análisis de la modalidad en las narrativas del cáncer puede revelar cómo las personas con cáncer crean un significado interpersonal en sus narrativas de salud, específicamente, cómo se posicionan para comunicar su experiencia a otras personas con cáncer. Esto ofrece información sobre cómo las personas con cáncer comparten sus puntos de vista con los demás que tienen como objetivo conectarse con otros, ofrecer consejos y reclamar conocimientos basados en sus experiencias. Dado que los hombres y las mujeres hablan sobre las enfermedades de manera diferente, la práctica médica puede beneficiarse de una comprensión de estas diferencias en determinados idiomas y culturas. Esta información puede ser relevante para hacer recomendaciones a los doctores, porque las personas responden mejor al tratamiento cuando se sienten comprendidas (Liu, Malin, Diamant, Thind \& Maly, 2013).

En este estudio, nos centramos en cómo tanto los hombres como las mujeres hablan en español sobre la leucemia y el linfoma de Hodgkin. Estos son cánceres que afectan a ambos géneros y sin las asociaciones de género de cáncer de mama o próstata. El análisis de estas narrativas es importante porque revela nuevas ideas sobre cómo las personas se comunican sobre sus experiencias colectivas de tener cáncer, incluido cómo los pacientes con cáncer enmarcan los sentimientos y las reacciones a la enfermedad (Skott, 2002). El estudiar narrativas en línea también contribuye a nuestro conocimiento de cómo los pacientes con cáncer discuten esta enfermedad en términos no literales en un idioma que no es el inglés. Aquí nos enfocamos en las metáforas que 
los participantes usaron para hablar sobre el cáncer y cómo transmitieron sus mensajes mediante el análisis de la modalidad.

\section{Marco teórico}

Según la teoría conceptual de la metáfora, la metáfora es un elemento central en la forma en que las personas piensan y expresan sus pensamientos en el lenguaje (Lakoff \& Johnson, 1980). Metáforas como 'El amor es una trayectoria,' conectan dos dominios, uno de los cuales (amor) es más abstracto que el otro (trayectoria). Las declaraciones incluyen 'Mira qué tan lejos hemos llegado', 'Toma mi mano y caminemos juntos' y 'Tendremos que ir por caminos separados'. Las personas usan metáforas para hablar sobre conceptos abstractos porque es más fácil entenderlos en relación a algo más concreto. El análisis de metáforas es útil para este estudio debido al papel de las metáforas en la construcción del significado y su realización a través de las elecciones de lenguaje. Estos significados nos dan una idea de las construcciones culturales de las condiciones de salud y revelan información ideológica.

Para estudiar la modalidad se utilizó una perspectiva de lingüística sistémica funcional (LSF). La modalidad dentro de la LSF se considera un componente de la metafunción interpersonal la cual se relaciona con las formas en que los hablantes expresan actitudes y juicios (Halliday \& Matthiessen, 2004). La modalidad consiste en la modalización (realizada con estructuras gramaticales para expresar probabilidad y frecuencia) y la modulación (realizada con estructuras gramaticales para expresar voluntad y obligación). La modalización se refiere a los significados modales utilizados para indicar diversos grados de proximidad entre los extremos binarios positivos y negativos, específicamente obligación y disposición (Lavid, Arús \& Zamorano, 2010). Los recursos gramaticales de la modalización también se pueden utilizar como atenuadores (Magaña, 2017).

Estructuralmente, la modalización se puede expresar de tres maneras posibles: operadores modales finitos (por ejemplo, 'poder'), adjuntos de modo (por ejemplo, 'posiblemente') y una combinación de ambos (por ejemplo, 'posiblemente puede') (Eggins, 2004). Estos incluyen los adjetivos, adverbios, sustantivos y verbos que expresan probabilidad. Los interlocutores pueden usar explícitamente los complementos de estado de ánimo con la modalidad de rango como 'supongo' (probabilidad baja), 'creo' (probabilidad mediana) y 'estoy seguro/a' (probabilidad alta) (Eggins, 2004). Las expresiones con alta probabilidad como en 'estoy seguro/a' se consideran modalizadas porque la proposición sería más cierta sin el adjunto modal. Además de estas opciones, Lavid et al. (2010) proponen que en español los verbos para expresar el futuro o el condicional crean modalización.

Gramaticalmente, los verbos modales en la cláusula proyectora que requieren la conjunción 'que' se consideran adjuntos modales, dado que funcionan fuera de la 
cláusula principal. Estos verbos modales también se consideran ejemplos de metáfora gramatical (de modalidad) ya que son cláusulas técnicamente completas (con su propia estructura MOOD / RESIDUE) (Halliday \& Matthiessen, 2004). Aún así, funcionan para modalizar la cláusula principal (Eggins, 2004); por ejemplo, 'pensar que' se puede reemplazar con 'es posible que' o 'posiblemente' y mantener el mismo impacto gramatical en la cláusula: 'pensaba que no salía de esa', 'es posible que no salga de esta' y 'posiblemente no salga de esta'. Si bien el impacto en la cláusula es similar, el significado subjetivo cambia ligeramente.

Este estudio se enfoca en un análisis de los verbos modales que expresan probabilidad y obligación, porque son recursos de modalidad más comunes no sólo en el español médico (Magaña, 2017), sino también en el español (y en el inglés) a través de distintos contextos (Hyland \& Milton, 1997; Martín Martín, 2000; Magaña, 2021). Por ejemplo, en su comparación de artículos de investigación en inglés y en español, Martín Martín (2000) encontró que los verbos modales son más comunes en ambas lenguas y que la modalidad expresada mediante recursos adverbiales (posiblemente, tal vez) y adjetivales (posible) tienen una baja frecuencia.

\section{Marco metodológico}

\subsection{Participantes/Corpus}

Para este estudio, analizamos 50 entradas generadas por sobrevivientes de cáncer y pacientes hispanohablantes en un sitio web en línea, http://wwwfcarrerasorg. En este foro, las víctimas de cáncer comparten sus sentimientos y experiencias personales con la enfermedad, incluidos los sentimientos sobre el proceso de tratamiento. También ofrecen consejos a otros. Las 50 narraciones fueron escritas en español. Hubo un total de 34.134 palabras (17.047 para las narraciones de los hombres y 17.087 para las narraciones de las mujeres) en nuestro corpus. Los contribuyentes fueron 25 hombres y 25 mujeres, todos hablantes nativos de español. ${ }^{1}$ La mayoría de estos individuos eran de España (46), pero algunos eran de Argentina y México. Todos eran adultos que habían estado lidiando o que actualmente padecían la leucemia o el linfoma de Hodgkin. Todas las entradas fueron producidas o actualizadas en $2016 .{ }^{2}$

\subsection{Procedimiento}

Después de crear el corpus, nos familiarizamos con las narraciones notando su contenido, significado, estilo y tono. Identificamos las descripciones metafóricas del cáncer al examinar cómo describieron las personas sus experiencias con esta enfermedad, incluidos sus sentimientos hacia los diagnósticos y el tratamiento, así como sus consejos a los demás. Seguimos el enfoque del Grupo Pragglejaz (2007) para el Procedimiento de identificación de metáforas (Metaphor Identification Procedure) (también vea Cameron \& Maslen, 2010). A pesar de algunas limitaciones, este enfoque 
es ampliamente utilizado y apropiado para identificar y analizar la metáfora en el discurso natural (para una discusión sobre el tema, vea Gibbs, 2017).

Nuestros datos se clasificaron en tres categorías generales de metáfora: (1) guerra, que presentaba un lenguaje que metafóricamente se refería a los enfrentamientos, como la pelea, la derrota y la victoria; (2) trayectoria, que metafóricamente se referían al movimiento, como caminos, carreras, trenes y obstáculos que impedirían el movimiento; y (3) otro tipo, que incluía un lenguaje metafórico que no encajaba en las otras dos categorías (por ejemplo, descripciones del cáncer como un objeto físico o como un juego de lotería). Para nuestro análisis, examinamos cómo la metáfora se refería al cáncer (por ejemplo, referencia a la condición misma, referencia al tratamiento). Nos enfocamos en frases que metafóricamente se referían a algún aspecto del cáncer. Cada vez que una frase dada obviamente incluía metáforas, se codificaba como una declaración metafórica. Por ejemplo, 'La enfermedad no pudo conmigo' fue codificada como un ejemplo de metáfora (metáfora de la guerra). En algunos casos, las declaraciones incluyeron dos metáforas diferentes, como en 'Se puede luchar y salir de esto’ (metáfora de guerra, metáfora de trayectoria). En otros, las declaraciones incluían dos instancias de la misma metáfora, como en 'Tengo que estar atento a la batalla y no bajar la guardia' (metáfora de guerra, metáfora de guerra).

La probabilidad y la obligación son particularmente útiles para el género que se investiga en este trabajo porque las narrativas sobre el cáncer se relacionan con la forma en que se presenta la información y el tipo de consejo que las personas con cáncer ofrecen a sus lectores. Aunque los verbos modales pueden tener múltiples usos, nos centramos en cuándo fueron utilizados para expresar la probabilidad u obligación. Por ejemplo, en el caso de 'poder', no codificamos aquellos que comunicaron capacidad (por ejemplo, 'puedo comer'), los cuales tienden ser parte del operador finito. Solo se analizaron los verbos modales que expresaron la posibilidad ('puede que funcione el tratamiento'), los cuales frecuentemente forman parte de cláusula proyectora con el uso de la conjunción 'que' (es decir, el adjunto modal) que funciona para modalizar la cláusula principal.

\section{Resultados}

Identificamos 341 frases metafóricas que se refieren al cáncer en el corpus. Nos centramos en las descripciones metafóricas del tratamiento del cáncer a lo largo de varias dimensiones, el enterarse de la enfermedad en sí misma y el papel del paciente a la hora de lidiar con el cáncer, el tratamiento y las secuelas asociadas con el cáncer. Como se muestra en el Gráfico 1, cuando las personas usaban metáforas para comunicarse sobre el cáncer, la mayoría de las veces usaban el lenguaje de la guerra. El lenguaje de trayectoria era menos común y otro lenguaje metafórico lo era aún menos. 
También encontramos que las mujeres usaban más metáforas que los hombres en todas las categorías.

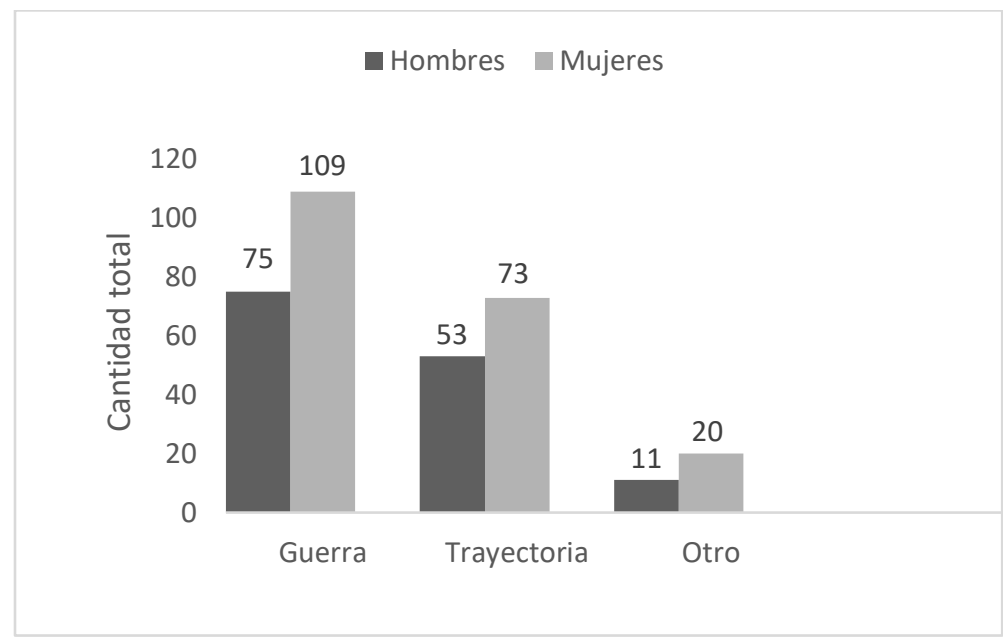

Gráfico 1. Uso de metáforas por hombres y mujeres.

La categoría de guerra incluye representaciones de cáncer como enemigo y el paciente como guerrero, referencias a animales depredadores, referencias a tener un ejército (formado por familia, equipo médico o partes del cuerpo / cuerpo) y referirse al tratamiento/medicamento como un arma. Las personas a menudo describieron el tratamiento del cáncer como una batalla que podría ganarse o perderse, como se ilustra en el ejemplo (1), donde una mujer de 20 años le dice a los lectores que deben luchar para ganar la batalla, y en el ejemplo (2), donde un hombre hace referencia a otras personas lidiando cáncer como compañeros de las tropas que han 'luchado a [su] lado' y han 'caído en el campo de batalla'.

(1) Con mi experiencia, me gustaría animar a todos aquellos que estéis pasando en este momento lo que yo viví hace un año, que, aunque haya momentos difíciles, en esta vida cuenta mucho cómo nos tomamos los problemas y tenemos que sacar una sonrisa y luchar por ganar la batalla. Mujer, 24 años.

(2) Dar gracias en especial, a todos aquellos que han luchado a mi lado y han caído en el campo de batalla. Hombre, 36 años.

Tanto hombres como mujeres usan metáforas de trayectoria para describir su experiencia con el cáncer, haciendo referencia a varios modos de viajar (por ejemplo, paseos en tren, carreras de pies, caminatas) y compañeros en su trayectoria. Muchos usaron la metáfora de trayectoria para animar a los lectores y hablar sobre el lado negativo de la enfermedad (por ejemplo, obstáculos). En el ejemplo (3), el escritor explica que la recuperación es un camino largo, pero que se mantiene firme sin detenerse. El escritor también describe a su esposa como una compañera en su trayectoria que lo ayuda a no caerse. 
(3) $\mathrm{Al}$ camino aún le queda un buen recorrido, pero avanzo con paso firme, sin prisas, pero sin pausas, de la mano de mi querida mujer, pieza fundamental para sostenerme cada vez que pueda tropezar y poder encontrar, por fin, el definitivo olvido de la enfermedad. Hombre, 54 años.

Las metáforas de trayectoria parecen una manera útil de pensar sobre el cáncer, porque, como en una trayectoria, hay un comienzo, un proceso y una llegada final a un destino. Cuando usan estas metáforas, los escritores se representan a sí mismos como viajeros, el tratamiento como una trayectoria y el destino como una cura. Los verbos de movimiento que se usan tienen una fuerza de resistencia que frena el movimiento, lo cual indica que se trata de una trayectoria difícil de cruzar, con obstáculos y con una meta que no siempre es alcanzable.

Encontramos que los escritores usaban verbos modales de forma estratégica cuando usaban metáforas del cáncer como 'tener que' en el primer ejemplo "tenemos que sacar una sonrisa y luchar para ganar la batalla" y 'poder' en el tercero "pueda tropezar". Los verbos modales más comunes eran 'creer que', 'haber que', 'pensar', 'poder que' y 'tener que'. Al hablar sobre el cáncer como un obstáculo, una joven usa la modalidad para aconsejar a sus lectores a que luchen y sigan adelante:

(4) [El cáncer] es un bache más que te pone la vida, innecesariamente y menos a estas edades, pero que una vez te lo ha puesto solo tienes que luchar y seguir adelante. Mujer, 21 años.

Tabla 1. Verbos modales usados por hombres y mujeres.

\begin{tabular}{|l|c|c|}
\hline & Hombres & Mujeres \\
\hline Creer que & $\mathbf{8}$ & $\mathbf{1 3}$ \\
\hline Deber & 3 & 4 \\
\hline Dudar & 2 & 2 \\
\hline Estar seguro/a de & 3 & 2 \\
\hline Haber que & 4 & $\mathbf{1 2}$ \\
\hline Imaginarse & 1 & 4 \\
\hline Necesitar & 11 & 8 \\
\hline Parecer que & 11 & 6 \\
\hline Pensar que & $\mathbf{1 0}$ & $\mathbf{2 3}$ \\
\hline Poder que & $\mathbf{2 1}$ & $\mathbf{3 2}$ \\
\hline Hacérsele que & 1 & 1 \\
\hline Sentir que & 3 & 1 \\
\hline Tener que & $\mathbf{2 0}$ & $\mathbf{1 3}$ \\
\hline \multicolumn{2}{|l}{} & \\
\hline Total & $\mathbf{9 8}$ & $\mathbf{1 2 1}$ \\
\hline
\end{tabular}

Una de las diferencias clave en el uso de verbos modales por género fue que las mujeres usan más procesos mentales ('creer' y 'pensar') que los hombres. ${ }^{3}$ Usan estos 
procesos mentales para expresar la probabilidad cuando ofrecen consejos y comparten experiencias íntimas. En (5), una mujer aconseja a sus lectores que durante el tratamiento del cáncer uno debe ser como un caballo, quedarse ciego y seguir mirando hacia adelante (metáfora de trayectoria). La autora usa 'creo que' para evitar comprometerse completamente con esta recomendación, tal vez debido a las connotaciones negativas de la ignorancia voluntaria. De manera similar, en (6), una escritora ofrece ánimo a sus lectores y les dice que luchen con uñas y dientes para superar la enfermedad. Luego mitiga el consejo diciendo que ella 'piensa' que una buena actitud es importante para la recuperación, tal vez reconociendo que esto presiona a las personas que sufren para que sean optimistas y/o que poca evidencia científica respalda esta opinión.

(5) Creo que en estos momentos uno debe hacer como los caballos; vendar sus ojos de costado y mirar siempre adelante. Mujer, 30 años.

(6) Animo a todas las personas que estéis atravesando estos duros momentos, a que luchéis por superar la enfermedad con uñas y dientes, poniendo mucho de vuestra parte, ya que creo que es fundamental el buen estado anímico para una mejor y más rápida recuperación. Mujer, 30 años.

Las personas con cáncer usan verbos modales con una modalidad alta para revelar sus vulnerabilidades, por ejemplo, admitir fallas. En (7), una mujer joven dice que ella era 'ingenua' al pensar que el cáncer no se podía superar. Ella usa otro verbo modal para asegurarles a sus lectores que es posible 'continuar'. En (8), de manera similar, otra mujer de 20 años admite que se equivocó al pensar que estaba viviendo uno de los mejores momentos de su vida y que una vez que descubrió que tenía cáncer tuvo que dejar todo de lado para comenzar su lucha.

(7) Era ingenua, lo admito, y no por no saber lo que me quedaba por sufrir, sino por pensar que el cáncer no se podía superar. Sin embargo, aquí estoy, la prueba evidente de que no es así, de que se puede_seguir adelante. Mujer, 26 años.

(8) Tengo 26 años, creía encontrarme en uno de mis mejores momentos y ... ahora, de repente, tengo que dejar todo de lado y empezar a luchar por mi vida. Mujer, 26 años.

Los escritores también usan 'poder' para añadir probabilidad a sus declaraciones. Las mujeres usan este recurso lingüístico con más frecuencia que los hombres. En (9), una mujer de 40 años explica que es posible que las personas hagan lo que pueden y que es posible vencer al 'bicho'. Tal vez dada la incertidumbre de una enfermedad como el cáncer, ella decide no ofrecer certeza de un resultado positivo, solo una posibilidad de uno. 
(9) Mis palabras quiero que sirvan para concienciar a todas las personas que hayan sido diagnosticadas ahora o estén empezando el tratamiento de que se puede poner de tu parte, se puede ganar a ese bicho combinando los avances médicos con tu actitud, tu alimentación y apoyándote en los que más te quieren. Mujer, 40 años.

En (10), un hombre de unos 40 años informa a sus lectores sobre la importancia de ser un donante de médula ósea, ya que puede salvar vidas o ayudar a alguien durante su tratamiento. En (11), un hombre de unos 30 años dice que el cáncer podría quitarle su cuerpo, pero no su alma. Aquí usa el tiempo futuro de 'poder' para hablar sobre la posibilidad de muerte por cáncer.

(10) Y con ese gesto se puede salvar una vida, o al menos, dar la oportunidad a alguien de que pueda salvarla, de que pueda seguir luchando por salvarla. Hombre, 41 años.

(11) Si algo tengo claro en esta vida, es que se podrá llevar mi cuerpo, pero no mi alma. Por lo tanto, jamás logrará vencerme. Hombre, 32 años.

Los hombres también usan los procesos mentales, pero con menos frecuencia que las mujeres. En (12), un hombre hace referencia a una metáfora de trayectoria dos veces en "pensaba que no salía de esa" y "mis ganas de vivir me sacaron poco a poco adelante". En (13), un hombre se refiere al tratamiento del cáncer como una batalla y dice que cree que esa actitud es importante. En (14), un hombre usa metáforas de la guerra para hablar sobre el cáncer con incertidumbre y usa una variedad de verbos modales para expresarlo. Utiliza 'sentir que', que funciona de manera similar a 'pienso que' en "Nunca he sentido que la guerra está ganada" y 'pensar que tengo que estar atento para la batalla'.

(12) Pasé unos días malísimos y pensaba que no salía de esa pero mis fuerzas y mis ganas de vivir me sacaron poco a poco adelante. Hombre, 33 años.

(13) Aquí, donde ya empiezas a librar la batalla mental, además de la física, creo que es muy importante la actitud, o por lo menos así fue en mi caso. Hombre, 39 años.

(14) Nunca he sentido que la guerra está ganada; soy más de pensar que todos los días tengo que estar atento para la batalla y bajar la guardia. Hombre, 26 años.

A través de estos recursos lingüísticos para expresar modalidad, los escritores en los ejemplos anteriores invitan al lector a su proceso de pensamiento y buscan conectarse con ellos al mostrar sus vulnerabilidades. El usar estos recursos lingüísticos es estratégico cuando se habla de cáncer, dado que es una enfermedad llena de 
incertidumbres. Los pacientes son cuidadosos cuando hablan de un resultado y aconsejan a otros. Al utilizar la modalidad, los escritores mitigan sus declaraciones, dejando abiertas otras posibilidades, pero aun así son capaces de ofrecer consejos y de tratar de formar una conexión con sus lectores.

Los hombres usan la modulación, específicamente los verbos modales 'tener que' y 'necesitar', con más frecuencia que las mujeres. Estas son formas altas de modulación utilizadas para motivar al oyente por medio de órdenes, para compartir información sobre algo que tuvieron que hacer durante el tratamiento, incluyendo cambios en sus esfuerzos emocionales. En (15), un hombre de unos 30 años escribe sobre decirse a sí mismo que el cáncer era una batalla que tenía que ganar para referirse al esfuerzo emocional que hizo. En (16), otro hombre les dice a sus lectores cómo deben abordar el cáncer usando los verbos modales 'deber' y 'tener'.

(15) Pero con un algo que encontré en mi interior y gracias al apoyo de la familia, la novia, mis amigos y los grandes profesionales que nos ayudan, saqué unas fuerzas increíbles y dije que esta batalla la tenía que ganar yo. Hombre, 36 años.

(16) Entendimos que no te debes preguntar el "por qué" estás enfermo de Hodgkin, sino preguntarte "para qué". Hay una razón de esta prueba que te toca vivir y la tienes que descubrir. Tienes que salir fortalecido. Hombre, 35 años.

En (17), un escritor usa una metáfora de trayectoria que comúnmente encontramos en el lenguaje cotidiano, 'salir'. Aquí el escritor le dice a su lector que no se asuste y les asegura que todo 'saldrá bien' usando un mandato directo "no tengáis miedo". Encontramos este consejo en un par de narraciones de hombres, pero no en las narraciones de las mujeres. La función de estas altas formas de modulación es dar recomendaciones u ofrecer consejos en un estilo más asertivo.

(17) No tengáis miedo, va a salir todo bien. Hombre, 33 años.

Las mujeres también dan órdenes, pero de una manera menos directa, incorporándose a sí mismas en una obligación compartida utilizando formas como 'haber que' en combinación con metáforas de trayectoria y guerra como en los ejemplos (18)-(20). En (18), la paciente usa una metáfora de trayectoria para explicar que 'pasar' por el cáncer es difícil. Su mensaje para los afectados y sus familias es, "hay que ser fuertes". De manera semejante, en el ejemplo (19), una escritora dice que tengamos fe, esperanza y fortaleza para no permitir que el cáncer nos venza. 
(18) Quiero recordar a todas las familias que pasen por esta situación que, aunque cueste, hay que ser fuertes, que la fortaleza lo es todo, para enfermos y familiares. Mujer, 20 años.

(19) Hay que tener Fe, esperanza y, sobre todo, mucha fuerza para no dejarse vencer. Mujer, 30 años.

(20) Hay que luchar hasta el final, somos más y más fuertes, así que mucho ánimo a todos. Mujer, 37 años.

Los hombres también usan este recurso lingüístico de manera parecida, pero en nuestra muestra solo lo usaron tres hombres (uno de ellos lo usó dos veces).

(21) No hay que perder la esperanza, hay que luchar cada día como si fuese el último y no dejar que la enfermedad ocupe tu mente. Hombre, 34 años.

En general, los escritores de cáncer usan la guerra y la trayectoria para infundir esperanza y ofrecer consejo a sus lectores. El enfoque que utilizan los hombres y las mujeres difiere, según revelan los recursos de modalidad que eligen. Se ha observado que los hombres usan órdenes directas que ellos validan, mientras que las mujeres atienden a la presentación de verdades absolutas y normas, de manera que la obligatoriedad está en el mensaje más que en el valor de quien habla.

\section{Discusión}

Trabajos previos, incluidos Gibbs y Franks (2002) y Semino, Demjén, Hardie, Payne y Rayson (2018), arrojan luz sobre cómo se usa la metáfora en el discurso del cáncer en inglés, especialmente sobre cómo se usan las metáforas de guerra y de trayectoria. Este estudio se basa en ese trabajo al examinar cómo usan los hispanohablantes la metáfora para hablar sobre el cáncer y revelar nuevas ideas sobre las diferencias de género. Codificamos nuestro corpus de narrativas de cáncer producido por 50 hispanohablantes para todas las metáforas relacionadas con el cáncer y calculamos el número de apariciones de cada una. Una contribución importante de este estudio es que pudimos identificar tipos prominentes de metáforas en narrativas de cáncer en español. Descubrimos que las mujeres y los hombres usaban metáforas, en su mayoría metáforas de la guerra, pero también metáforas de trayectoria. Observamos que los pacientes usaban metáforas de la guerra para ofrecer consejos a los lectores y, a menudo retrataban al cáncer como un enemigo malvado mientras se presentaban como guerreros. Los pacientes tienden a personificar el cáncer y lo presentan como un enemigo de los escritores y lectores. También describían a su equipo médico, familia o cuerpo como un ejército, y aludían al tratamiento, la medicación y el cuidado de sí mismos como si fueran armas. 
Algunas metáforas son casi universales, sin embargo, cada idioma y cultura varía en términos de qué metáforas se utilizan, cómo se utilizan y cómo influyen en el pensamiento cotidiano sobre cuestiones importantes, incluida la enfermedad (Núñez \& Cornejo, 2012; Kövecses, 2015; Winter \& Matlock, 2017). Nuestro hallazgo de que las metáforas de la guerra predominan está en línea con Semino et al. (2015), quienes encontraron un efecto similar entre los pacientes con cáncer de habla inglesa. La similitud entre nuestros hallazgos y los de investigaciones anteriores sugiere que los hablantes de inglés y español conceptualizan el cáncer de manera semejante dada la presencia de la conceptualización de amplia difusión del dominio común de guerra y trayectoria (Lakoff \& Johnson, 1980).

Notamos que los escritores usaron metáforas de trayectoria para discutir su progreso y esperanzas y para ofrecer aliento a sus lectores. Utilizaron la metáfora de la trayectoria para enfatizar que el cáncer tiene un final. Al describir el cáncer como una trayectoria, las personas con cáncer hablaron sobre el camino a la recuperación y también explicaron las dificultades a las que se enfrentaron.

Muchas de estas referencias representaban la reflexión de los escritores sobre su experiencia general con el cáncer. Estas construcciones metafóricas nos dan una idea de las opciones lingüísticas dentro de un grupo social que hacen los escritores cuando comparten sus experiencias. Los escritores las utilizan para alinearse con un grupo interno y atraer a su audiencia (en particular a aquellos que también sufren de cáncer) mediante el uso de estas metáforas recurrentes (Magaña \& Matlock, 2018).

Descubrimos que las mujeres tienden a usar metáforas contra el cáncer con más frecuencia que los hombres. Una razón podría ser que las mujeres están más incrustadas en la comunidad del discurso del cáncer que los hombres. Consideramos que la contribución de las narrativas de cáncer a un foro público es una señal de esta incrustación. El sitio web que estudiamos tiene más contribuciones de mujeres que de hombres, y este hallazgo se alinea con investigaciones anteriores (Kim \& Chung, 2007).

El análisis de los usos de metáforas conceptuales nos brinda información sobre recursos lingüísticos para hablar indirectamente sobre el cáncer, mientras que el análisis de los verbos modales que se usan en estas metáforas nos ofrece una visión aún más detallada de los dispositivos gramaticales para expresar la probabilidad (lenguaje indirecto) y la obligación, revelando más diferencias preliminares de género en el discurso de cáncer. Encontramos que los verbos modales aparecían estratégicamente con las metáforas de guerra y de trayectoria. Al usar la modalidad, los hablantes demuestran su actitud sobre la probabilidad de su proposición. Debido a las incertidumbres de una enfermedad como el cáncer, la modalidad de estudio revela información importante acerca de cómo se entiende. En general, las mujeres usan más verbos modales que los hombres, sin embargo, difieren en los tipos de verbos 
modales que eligen. Las mujeres usan más expresiones de probabilidad a través de procesos mentales ('creo' y 'pensaba') que los hombres. Estos verbos modales funcionan para expresar duda y mostrar vulnerabilidad. Además, estos procesos mentales se usan de manera estratégica en casos en los que los escritores deciden no comprometerse completamente con sus declaraciones. Las mujeres también usaron estos recursos lingüísticos para compartir información o sentimientos personales. El uso de estos dispositivos de modalidad en las narrativas del cáncer puede ser un indicador de lo difícil que es hablar de problemas de salud relacionados con el diagnóstico y el tratamiento del cáncer.

Nuestro estudio está en línea con las investigaciones sobre angloparlantes que muestra que las mujeres en el discurso sobre el cáncer se preocupan principalmente por establecer conexiones con otras personas, mientras que los hombres se decantan por el intercambio o la búsqueda de información (Gooden \& Winefield, 2007; Kiss \& Meryn, 2001; Klemm et al., 1999; Seale, 2005; Seale et al., 2006). Encontramos que tanto la frecuencia como los tipos de verbos de modalidad que usan las mujeres y los hombres evidencian la inclinación de las mujeres a establecer conexiones y la tendencia de los hombres a ofrecer y buscar información. Al utilizar con frecuencia los procesos mentales para expresar vacilación, las mujeres mitigan las señales de que se consideran autoritativas e invitan a sus lectores a su proceso de pensamiento. Los hombres, por otro lado, comparten información sobre el cáncer con sus lectores dándoles consejos y órdenes directas. Los hombres acuden más a la modulación, específicamente, los procesos de tipo obligación, para dar órdenes, lo que los hace parecer más autoritarios. Las mujeres tienden a dar órdenes de forma indirecta mediante el uso de 'haber que' expresando subjetividad mientras que los hombres no se incluyen en la conceptualización y ofrecen su aserción como alternativa para el oyente utilizando órdenes directas.

\section{CONCLUSIONES}

La contribución de este estudio ha sido ofrecer un análisis cualitativo sobre los recursos lingüísticos que se usan en el discurso de cáncer en español y señalando diferencias preliminares de género. Este estudio tiene varias limitaciones, incluido el tamaño de la muestra. La investigación futura podría usar un corpus más grande y usar análisis estadístico para corroborar los hallazgos sugeridos aquí. Aún más, los estudios futuros centrados exclusivamente en la modalidad tienen el potencial de dar cuenta de todos los tipos de modalidad (y no solo los verbos modales como se ha hecho en este artículo). Un análisis sobre la modalidad en el discurso del cáncer puede brindar a los lingüistas la posibilidad de descubrir diferencias de género a niveles más detallados, por ejemplo, comparar expresiones de probabilidad y obligación entre hombres y mujeres (los niveles de asertividad, las diferencias en el uso de la segunda persona dirigidos al oyente). El enfoque únicamente en la modalidad también permitiría 
analizar los verbos modales según su impacto positivo y negativo para determinar en qué contextos los verbos como 'poder' y 'haber que' se usan para empoderar al paciente y en qué otros se perfila la potencialidad letal del cáncer. Otra línea de investigación sería un análisis enfocado en las metáforas conceptuales para relevar las subcategorías presentes dentro de las categorías predominantes de guerra y trayectoria.

Nuestros hallazgos de que los hispanohablantes se expresan de manera parecida a los angloparlantes en el uso de metáforas se pueden utilizar para ayudar a los miembros de la familia, amigos y compañeros de trabajo a encontrar formas más efectivas de comunicarse y pensar sobre el cáncer y brindar apoyo emocional. El cáncer es una enfermedad muy compleja. Los pacientes se enfrentan a una gran incertidumbre sobre el pronóstico, y las secuelas de la enfermedad y los tratamientos, incluida la quimioterapia, lo cual puede ser emocional y físicamente devastador para los pacientes y sus seres queridos. Esperamos que este estudio sobre cómo se comunican sobre su situación los pacientes de cáncer y los supervivientes conduzca a una mejor comprensión de cómo se conceptualiza y maneja la enfermedad, mejorando las interacciones médico-paciente, incluido el modo de discutir los planes de tratamiento y de diagnóstico y el papel de las personas que prestan apoyo a los pacientes.

\section{REFERENCIAS BIBLIOGRÁFICAS}

Achugar, M. (2008). Counter-hegemonic language practices and ideologies: Creating a new space and value for Spanish in Southwest Texas. Spanish in Context, 5(1), 1-19.

Bowker, J. (1996). Cancer, individual process, and control: A case study in metaphor analysis. Health Communication, 8(1), 91-104.

Brookes, D. \& Etkina, E. (2007). Using conceptual metaphor and functional grammar to explore how language used in physics affects student learning. Physical Review Special Topics - Physics Education Research, 3(1), 010105.

Caffi, C. (1999). On mitigation. Journal of Pragmatics, 31(7), 881-909.

Caffi, C. (2007). Mitigation. Ámsterdam: Elsevier.

Cameron, L. \& Maslen, R. (Eds.) (2010). Metaphor analysis: Research practice in applied linguistics, social sciences and the humanities. Londres: Equinox.

Casarett, D., Pickard, A., Fishman, J. M., Alexander S. C., Arnold, R. M., Pollak, K. I. \& Tulsky, J. A. (2010). Can metaphors and analogies improve communication with seriously ill patients? Journal of Palliative Medicine, 13, 255-260.

Charteris-Black, J. \& Seale, C. (2010). Gender and the language of illness. Nueva York: Palgrave Macmillan. 
Clark, H. H. (1973). Space, time, semantics, and the child. En T. Moore (Ed.), Cognitive development and the acquisition of language (pp. 27-63). Nueva York: Academic Press.

Cordella, M. (2007). 'No, no I haven't been taking it doctor': Compliance, face threatening acts and politeness in medical consultations. En M. E. Placencia \& C. García (Eds.), Linguistic politeness in the Spanish-speaking world (pp. 191-212). Mahwah, NJ: Lawrence Erlbaum.

Delbene, R. (2004). The function of mitigation in the context of a socially stigmatized disease: A case study in a public hospital in Montevideo, Uruguay. Spanish in Context, 1, 241-267.

Domínguez, M. \& Sapiña, L. (2016). Cancer metaphors in sports news: The match that must be won. En P. Ordoñez-López \& N. Edo-Marzá (Eds.), Medical discourse in professional, academic and popular settings (pp. 149-172). Bristol, UK: Multilingual Matters.

Duarte, M. (2016). Funciones estratégicas de las redes de metáforas en torno a 'socialismo': Un análisis interaccional del discurso político de Hugo Chávez. Revista Signos. Estudios de Lingüística, 49(90), 24-47 [en línea]. Disponible en: https://dx.doi.org/10.4067/S0718-09342016000100002.

Eggins, S. (2004). An introduction to systemic functional linguistics (2a edición). Londres: Continuum.

Epstein, A. S., Prigerson, H. G., O’Reilly E. M. \& Maciejewski, P. K. (2016). Discussions of life expectancy and changes in illness understanding in patients with advanced cancer. Journal of Clinical Oncology, 34(20), 2398-2403.

Flores-Ferrán, N. (2010). An examination of mitigation strategies used in Spanish psychotherapeutic discourse. Journal of Pragmatics, 42, 1964-1981.

Flores-Ferrán, N. (2012). Pragmatic variation in therapeutic discourse: An examination of mitigating devices employed by Dominican female clients and a Cuban American therapist. En J. C. Félix Brasdefer \& D. A. Koike (Eds.), Pragmatic variation in first and second language contexts: Methodological issues; Impact: studies in language and society (pp. 81-112). Filadelfia: John Benjamins.

Gibbs, R. W. (1994). The poetics of mind: Figurative thought, language, and understanding. Cambridge, UK: Cambridge University Press.

Gibbs, R. W. (2017). Metaphor wars: Conceptual metaphors in human life. Cambridge, UK: Cambridge University Press. 
Gibbs, R. W. \& Franks, H. (2002). Embodied metaphor in women's narratives about their experiences with cancer. Health Communication, 14(2), 139-165.

Gooden, R. J. \& Winefield, H. R. (2007). Breast and prostate cancer online discussion boards: A thematic analysis of gender differences and similarities. Journal of Health Psychology, 12(1) 103-114.

Grupo Pragglejaz (2007). MIP: A method for identifying metaphorically used words in discourse. Metaphor and Symbol, 22(1), 1-39.

Ha, J. F. \& Longnecker, N. (2010). Doctor-patient communication: A review. The Ochsner Journal, 10(1), 38-43.

Halliday, M. A. K. \& Matthiessen, C. M. I. M. (2004). An introduction to functional grammar (3a edición). Londres: Edward Arnold.

Harrison, J., Maguire, P. \& Pitceathly, C. (1995). Confiding in crisis: Gender differences in pattern of confiding among cancer patients. Social Science \& Medicine, 41(9), 1255-1260.

Henderson, W. (1982). Metaphor in economics. Economics, 18(4), 147-153.

Hurley, K. E. (2014). To fight, or not to fight: A cancer psychotherapist with cancer confronts the battle metaphor. Women \& Therapy, 37(3-4), 311-318.

Hyland, K. (1996). Writing without conviction? Hedging in science research articles. Applied Linguistics, 17(4), 433-454.

Hyland, K. \& Milton, J. (1997). Qualification and certainty in L1 and L2 students' writing. Journal of Second Language Writing, 6(2), 183-205.

Kim, S. \& Chung, D. S. (2007). Characteristics of cancer blog users. Journal of the Medical Library Association: JMLA, 95(4), 445-450.

Kiss, A. \& Meryn, S. (2001). Effect of sex and gender on psychosocial aspects of prostate and breast cancer. British Medical Journal, 323, 1055-1058.

Klemm, P., Hurst, M., Dearholt, S. L. \& Trone, S. R. (1999). Gender differences on Internet cancer support groups. Computers in Nursing, 17(2), 65-72.

Koller, V. (2004). Businesswomen and war metaphors: 'Possessive, jealous and pugnacious'? Journal of Sociolinguistics, 8(1), 3-22.

Kövecses, Z. (2003). Metaphor and emotion: Language, culture, and body in buman feeling. Cambridge, UK: Cambridge University Press.

Kövecses, Z. (2005). Metaphor in culture: Universality and variation. Cambridge, UK: Cambridge University Press. 
Kövecses, Z. (2008). Conceptual metaphor theory: Some criticisms and alternative proposals. Annual Review of Cognitive Linguistics, 6(1), 168-184.

Kövecses, Z. (2015). Where metaphors come from: Reconsidering context in metaphor. Nueva York: Oxford University Press.

Lakoff, G. (2008). The political mind: Why you can't understand 21st-century politics with an 18th-century brain. Nueva York: Penguin Books.

Lakoff, G. \& Johnson, M. (1980). Metaphors we live by. Chicago: University of Chicago Press.

Lakoff, G. \& Núñez, R. (2000). Where mathematics come from: How the embodied mind brings mathematics into being. Nueva York: Basic Books.

Laranjeira, C. (2013). The role of narrative and metaphor in the cancer life story: A theoretical analysis. Medine Health Care and Philosophy, 16, 469-481.

Lavid, J., Arús, J. \& Zamorano, J. R. (2010). Systemic functional grammar of Spanish: A contrastive study with English. Londres: Continuum.

Liu, Y., Malin, J. L., Diamant, A. L., Thind, A. \& Maly, R. (2013). Adherence to adjuvant hormone therapy in low-income women with breast cancer: The role of provider-patient communication. Breast Cancer Research Treatment, 137(3), 829-836.

Magaña, D. (2017). Modality resources in Spanish during psychiatric interviews with Mexican patients. Communication \& Medicine (2016), 13(3), 291-305.

Magaña, D. (2021). Modality across genres in Spanish as a heritage language. Revista Española de Lingüistica Aplicada.

Magaña, D. \& Matlock, T. (2018). How Spanish speakers use metaphor to describe their experiences with cancer. Discourse \& Communication, 12(6), 627-644.

Martín Martín, P. (2000). Epistemic modality in English and Spanish psychological texts. LFE. Revista de Lenguas para Fines Especificos, 7-8, 196-208.

Matlock, T. (2013). Motion metaphors in political races. En M. Borkent, N. Dancygier \& J. Hinnell (Eds.), Language and the Creative Mind (pp. 193-201). Stanford, CA: CSLI Publications.

Matlock, T., Castro, S. C., Fleming, M., Gann, T. M. \& Maglio, P. P. (2014). Spatial metaphors of web use. Spatial Cognition \& Computation, 14(4), 306-320.

Moore, K. E. (2006). Space-to-time mappings and temporal concepts. Cognitive Linguistics, 17, 199-244. 
Núñez, R. \& Cornejo, C. (2012). Facing the sunrise: Cultural worldview underlying intrinsic-based encoding of absolute frames of reference in Aymara. Cognitive Science, 1-27.

Raison, C. L. \& Miller, A. H. (2003). Depression in cancer: New developments regarding diagnosis and treatment. Biological Psychiatry, 54(3), 283-294.

Reisfield, G. \& Wilson, G. (2004). Use of metaphor in the discourse on cancer. Journal of Clinical Oncology, 22(19), 4024-4027.

Rojas Miranda, D. \& Fernández González, L. (2015) ¿Contra qué se lucha cuando se lucha? Implicancias clínicas de la metáfora bélica en oncología. Revista Médica de Chile, 143(3), 352-357.

Santa Ana, O. (2002). Brown tide rising: Metaphors of Latinos in contemporary American public discourse. Austin: University of Texas Press.

Seale, C. (2005). New directions for critical internet health studies: Representing cancer experience on the web. Sociology of Health and Illness, 27(4), 515-540.

Seale, C., Ziebland, S. \& Charteris-Black, J. (2006). Gender, cancer experience and internet use: A comparative keyword analysis of interviews and online cancer support groups. Social Science \& Medicine, 62, 2577-2590.

Semino, E., Demjén, Z., Demmen, J., Koller, V., Payne, S., Hardie, H. \& Rayson, P. (2015). The online use of 'violence' and 'journey' metaphors by patients with cancer, as compared with health professionals: A mixed methods study. BMJ Supportive and Palliative Care, 7, 60-66. Doi:101136/bmjspcare-2014-000785.

Semino, E., Demjén, Z., Hardie, A., Payne, S. \& Rayson, P. (2018). Metaphor, cancer and the end of life: A corpus-based study. Nueva York: Routledge.

Skott, C. (2002). Expressive metaphors in cancer narratives. Cancer Nursing, 25(3), 230235.

Sontag, S. (1989). AIDS and its metaphors. Londres: Allen Lane.

Stark, D. P. H. \& House, A. (2000). Anxiety in cancer patients. British Journal of Cancer, $83(10), 1261-1267$.

Thibodeau, P. H. \& Boroditsky, L. (2011). Metaphors we think with: The role of metaphor in reasoning. PLOS ONE, 6(2), e16782.

Williams, J. (2016). Get the metaphor right! Cancer treatment metaphors in the English and Spanish press. Alfinge, 28, 109-138.

Winter, B. \& Matlock, T. (2013). Reasoning about similarity and proximity. Metaphor \& Symbol 28, 1-14. 


\section{* AGRADECIMIENTOS}

Agradecemos a los miembros del grupo de investigación Matriz de Ciencias Sociales de UC Berkeley por muchas discusiones útiles sobre metáforas del cáncer, especialmente a Oana David y Eve Sweetser. Agradecemos a los estudiantes de UC Merced por su apoyo en codificar datos, especialmente a Gloria Quintana y Mauricio Martínez. Agradecemos las sugerencias constructivas de una versión previa de los revisores anónimos. Finalmente, agradecemos los útiles comentarios de Jorge Arús Hita y Nora Kaplan y sus esfuerzos en organizar esta colaboración.

\section{NOTAS}

${ }^{1}$ En Magaña y Matlock (2018), usamos las mismas 25 narrativas escritas por mujeres.

${ }^{2}$ Los administradores del sitio web nos concedieron permiso para usar las narrativas para este estudio.

${ }^{3}$ Notemos que aunque algunos de los verbos modales son procesos mentales, los verbos modales también se expresan mediante otros procesos (e.g., materiales, de comportamiento, relacionales). 